\title{
Effect of turning vs. supine position under phototherapy on neonates with hyperbilirubinemia: a systematic review
}

\author{
Shalin Lee Wan Fei and Khatijah L Abdullah
}

\begin{abstract}
Aims and objectives. To determine the most effective position jaundiced neonates should assume during phototherapy from appraised randomised controlled trials.

Background. Many local hospitals still alternate positions of jaundiced neonates receiving phototherapy despite the safe infant sleeping protocol of placing them supine.

Design. A systematic review was conducted.

Methods. Databases that included Cumulative Index to Nursing and Allied Health Literature, ScienceDirect, Embase and The Cochrane Library were used. Randomized controlled trials published in English language that evaluate the best position for healthy jaundiced neonates aged day 1 to 14 under phototherapy were searched. In addition, any positioning done every 2-3 hours during phototherapy with the outcome measures being bilirubin reduction and duration of phototherapy were also searched and included $(n=5)$. Physiotherapy Evidence Database scale was adopted for quality assessment. All processes were conducted by both reviewers independently. Discrepancies were resolved by a third reviewer. Preferred Reporting Items for Systematic Reviews and MetaAnalyses Guideline were utilised. Out of 20 papers, five were included for qualitative synthesis. Data extraction was based on the template (participants, study designs etcetera) agreed by both authors.

Results. All five studies possessed external validity. One paper scored 7, three scored 5 while one scored 3. Four of these studies reported no difference in bilirubin reduction and duration of phototherapy. Only one study reported a significant drop in serum bilirubin and shorter duration of phototherapy in the supine group.

Conclusion. It has been proved that keeping the jaundiced newborns in the supine position throughout phototherapy is as effective as turning them periodically based on the appraised studies.
\end{abstract}

What does this paper contribute to the wider global clinical community?

- Keeping jaundiced neonates in the supine position throughout phototherapy is as effective as turning them periodically based on the reported appraised trials.

- Nurses' workload will be lightened as it is unnecessary to alternate the positions of the jaundiced neonates two to three hourly when conventional phototherapy is delivered.

\footnotetext{
Authors: Shalin Lee Wan Fei, Master of Nursing Science Candidate, Bachelor of Nursing with Honours, Certificate in Paediatric Nursing, Pediatric Advanced Life Support (PALS) Provider, Registered Nurse, Department of Nursing Science, Faculty of Medicine, University Malaya, Kuala Lumpur, Wilayah Persekutuan, Malaysia; Khatijah L Abdullah, Bachelor of Science in Nursing Studies, Masters of Science in Health Services Management, Doctorate in Clinical Practice (Nursing), Midwifery, Registered Nurse, Department of Nursing Science, Faculty of Medicine, University Malaya, Kuala Lumpur, Wilayah Persekutuan, Malaysia
}

Correspondence: Shalin Lee Wan Fei, Lecturer, Department of Nursing, Faculty of Medicine and Health Sciences, University Malaysia Sarawak, Jalan Datuk Mohd Musa, 94300 Kota Samarahan, Sarawak, Malaysia. Telephone: +6 082581000 (ext. 5677) E-mail: lwfshalin@gmail.com.

Source of support: PPP UM Grant P0038-2013B 
Relevance to clinical practice. It is unnecessary to alternate positions of the jaundiced neonates when conventional phototherapy is delivered to lighten nurses' workload.

Key words: alternate, hyperbilirubinemia, jaundice, neonatal, newborn, phototherapy, position, total serum bilirubin level, turn

Accepted for publication: 2 August 2014

\section{Introduction}

\section{Background}

Neonatal jaundice (NNJ) is a common condition among newborns throughout the world. More than half of term and preterm neonates developed NNJ in their first week of life (Welsh 2010). The yellowing of the skin and organs in physiological jaundice is caused by circulating unconjugated bilirubin produced by haemolysis of fetal erythrocytes. Furthermore, functions of the neonatal liver have yet to be at its optimum to metabolise the unconjugated bilirubin. Nevertheless, there are also rare instances involving pathological jaundice such as blood group incompatibility, haemolysis, gastrointestinal obstruction and other causative factors.

Possible progression of NNJ without appropriate intervention may lead to severe and irreversible neurological threat. Therefore, according to the Paediatric Protocols for Malaysian Hospitals, treatment options such as conventional phototherapy, intensive phototherapy or exchange transfusion remain the mainstay based on the criteria and assessments done (Ismail et al. 2012).

The debates on whether to place the jaundiced neonates in a supine or alternate position throughout the course of phototherapy were attributed to the differing conclusions drawn by different researchers from decades ago to the 21st century. Based on the arguments by Vogl et al. (1978) and Lau and Fung (1984), theoretically, the blanched surface of a jaundiced newborn on phototherapy would be reloaded with bilirubin when the exposed surface has been changed and alternated with the bilirubin-loaded side. Based on their models, changing the position of jaundiced neonates or implementing intermittent phototherapy will hasten the drop of total serum bilirubin (TSB) by optimising photoisomerisation. Nevertheless, the outcomes of several clinical trials did not support this biological concept and have proven otherwise. As a result, these clinical trials will be appraised and evaluated for their quality and reliability.

This systematic review is the first to examine the most effective position for jaundiced neonates who were nursed under phototherapy. This review is significant because turning a child every two hour used to be one of the nursing interventions delivered to neonates who underwent phototherapy in some local hospitals in Malaysia. Although guidelines such as Paediatric Protocol for Malaysian Hospital (3rd ed.) (Ismail et al. 2012) have removed the two-hourly turning in the care of neonates under phototherapy in the latest edition, coupled with the National Institute for Health and Care Excellence (NICE) (2010) and Queensland Maternity and Neonatal Clinical Guidelines Program (2009) of placing newborns supine under phototherapy at all times according to the safe infant sleeping protocol, many hospitals still alternate the positions of these jaundiced neonates receiving phototherapy as reported by Mohammadzadeh et al. (2004) and Hansen (2012). This systematic review is therefore imperative in drawing the attention of nurses and nurse managers who are still alternating the positions of neonates with hyperbilirubinemia two to three hourly when phototherapy is delivered.

\section{Objective}

The objective of this systematic review is to determine the most effective position jaundiced neonates should assume during phototherapy in bringing the TSB level down to the normal range from appraised randomised controlled trials (RCTs). Studies have been conducted to explore the most effective treatment in preventing the development of severe NNJ, ranging from the invasive to the noninvasive therapy. Subsequent to the appraisal, the outcomes generated from these trials will be able to give light to the necessity of frequent turning during phototherapy for NNJ. Frequent turning refers to the changing of positions as often as every two to three hours. 


\section{Methods}

\section{Search strategy}

The search process was conducted by both the review authors independently. Discrepancies were brought forward to be discussed. If agreement was not reached, it has to be resolved by a third reviewer. Nonetheless, there was no disagreement between both the review authors in this systematic review. Randomized controlled trials (RCTs) that are related to the most effective position during phototherapy for NNJ had been searched through electronic databases via the University of Malaya's interactive webpage. Databases that included Cumulative Index to Nursing and Allied Health Literature (CINAHL), ScienceDirect, Embase and The Cochrane Library were utilised in the search for sources to meet the criteria. Keywords, MeSH terms and their synonyms (example, jaundice also means hyperbilirubinemia or icterus), in combination with the other subject headings using operators such as truncations, wildcards, Boolean searching ("AND" and "OR") as specified in Table 1, were fed into the search engine to generate more specific hits which are pertinent to the objective. Different databases have different operators and approaches. For example, in ScienceDirect, the search strategy of "("neonatal jaundice" OR "icterus neonatorum" OR "newborn jaundice" OR "neonatal hyperbilirubin\#emia" OR "newborn hyperbilirubin\#emia") AND photo therapy AND ("bilirubin level" OR "total serum bilirubin" OR "serum bilirubin") AND ("position*” OR "turn*” OR “alternate”)" OR (MM “Jaundice, Neonatal/TH")" was fed into the search engine. The operators such as " " denotes phrase, \# means alternate spelling that may contain an extra character and * signifies root word that may end with anything. The search was further refined by restricting to only full text, age group from birth to 1 month, English language and RCTs. Additionally, the search for relevant articles was specifically filtered from the year 1991-2013 due to the fact that improvements in reporting RCTs had only begun to gather momentum in the mid-1990 (Schulz et al. 2010). With that, a total of 19 hits had been obtained from all the databases. The quest resumed by going through the reference lists of the retrieved papers for relevant journal articles.

\section{Inclusion criteria}

Randomised controlled trials that evaluate the best position under phototherapy for NNJ were searched and included for review. Healthy preterm and term neonates with postnatal age more than 24 hours and within the first two weeks of life who developed physiological hyperbilirubinemia were sought. Neonates with blood group and Rhesus incompatibility, G6PD deficiency, haemolysis of other causes, sepsis and gastrointestinal obstruction were excluded. The positions of neonates nursed under phototherapy for NNJ have been evaluated. Phototherapy denotes treatment under lamps emitting light energy of appropriate spectrum, wavelength, distance and irradiance. It is able to convert indirect bilirubin in the skin to the harmless water-soluble bilirubin to be excreted in the urine (Welsh 2010). Positions under review may include supine or prone position done every two to three hours during phototherapy. The primary outcome measures include the TSB level reduction. Meanwhile, the secondary outcome measure evaluates duration of therapy (in hours) for TSB level to fall within the normal range. Articles written or translated into English were included. The related research articles published in languages other than English were subsequently excluded from the review.

Table 1 Search strategies

\begin{tabular}{|c|c|}
\hline Database & Search terms \\
\hline CINAHL & $\begin{array}{l}\text { (“neonatal jaundice” OR “icterus neonatorum” OR “newborn jaundice” OR “neonatal hyperbilirubin\#emia” } \\
\text { OR “newborn hyperbilirubin\#emia”) AND photo therapy AND (“bilirubin level” OR "total serum bilirubin” } \\
\text { OR “serum bilirubin”) AND (“position*” OR “turn*” OR “alternate”)" OR (MM “Jaundice, Neonatal/TH”) }\end{array}$ \\
\hline ScienceDirect & $\begin{array}{l}\text { (\{neonatal jaundice }\} \text { OR }\{\text { icterus neonatorum\} OR }\{\text { newborn jaundice }\} \text { OR \{neonatal hyperbilirubin*emia }\} \text { OR } \\
\text { \{newborn hyperbilirubin*emia\}) AND (phototherapy) AND (turn OR position OR alternate) AND (\{bilirubin level\} } \\
\text { OR \{total serum bilirubin\} OR \{serum bilirubin\}) }\end{array}$ \\
\hline Embase & $\begin{array}{l}\text { ('neonatal jaundice'/exp OR 'icterus neonatorum'/exp OR 'newborn jaundice'/exp OR 'neonatal hyperbilirubin?emia' } \\
\text { OR 'newborn hyperbilirubin?emia') AND phototherapy/exp AND (turn* OR position* OR alternate) AND } \\
\text { ('bilirubin level' OR 'total serum bilirubin' OR 'serum bilirubin'/exp) }\end{array}$ \\
\hline Cochrane Library & $\begin{array}{l}\text { "neonatal jaundice” OR “icterus neonatoturm” OR “newborn jaundice” OR "neonatal hyperbilirubin?emia” OR } \\
\text { "newborn hyperbilirubin?mia” AND phototherapy and position* OR turn* OR alternate AND "bilirubin level” } \\
\text { OR "total serum bilirubin" OR "serum bilirubin" }\end{array}$ \\
\hline
\end{tabular}

CINAHL, Cumulative Index to Nursing and Allied Health Literature. 


\section{Screening for eligibility}

The title and abstracts of the retrieved papers have been scanned through and later, the full texts sieved through. Journals which have been excluded were papers which were not specific to NNJ, the design was not RCT, the interventions and comparisons were not related to positioning of neonates under phototherapy.

\section{Data extraction}

Study selection, quality assessments and data extraction have been conducted by both the reviewers independently. Discrepancies have to be resolved by a third reviewer. Nonetheless, outcomes and conclusion drawn from both the review authors in this systematic review were similar. The selection was based on the Inclusion criteria stated. Study selection that started with identification, screening for eligibility and inclusion was based on the step-by-step guideline by Preferred Reporting Items for Systematic Reviews and Meta-Analyses (PRISMA) 2009 Flow Diagram by Moher et al. (2009). Figure 1 illustrates the selection of studies. Out of the hits obtained from the search strategies in the databases, a total of 19 related records were identified from CINAHL $(n=3)$, ScienceDirect $(n=3)$, Embase $(n=10)$ and The Cochrane Library $(n=3)$. Review of the reference lists of selected articles retrieved two associated papers. The screening resulted in the removal of one article leaving only 20 studies to be screened. Primary screening from the title and abstract has excluded seven articles because these studies were comparing other treatments $(n=2)$, measuring other outcomes $(n=3)$, not available in English language $(n=1)$ and not specific to NNJ $(n=1)$. Later, the 13 full-text articles were assessed for eligibility in accordance to the Inclusion criteria described. A total of eight papers were excluded for being non-RCT $(n=5)$ and for comparing other treatments $(n=3)$. Consequently, five papers were included in the qualitative synthesis.

Data extracted from each research article was based on a template agreed upon between both the review authors. The parameters contained in the template include quality of the respective study, baseline and demographic information, confounding factors, participants, study designs, methods, findings and conclusion, which will be described in the subsequent sections.

\section{Quality assessment}

In evaluating clinical trials, an appropriate tool must be selected. In this systematic review, the Physiotherapy Evidence Database (PEDro) scale, which is a comprehensive and reliable scale that consists of the widely used two-item Jadad scale and the nine-item Delphi list (Maher et al. 2003), has been used to evaluate the quality of each RCT paper reviewed. This scale was opted for because it has been concluded by Maher et al. (2003) that it is a reliable tool to

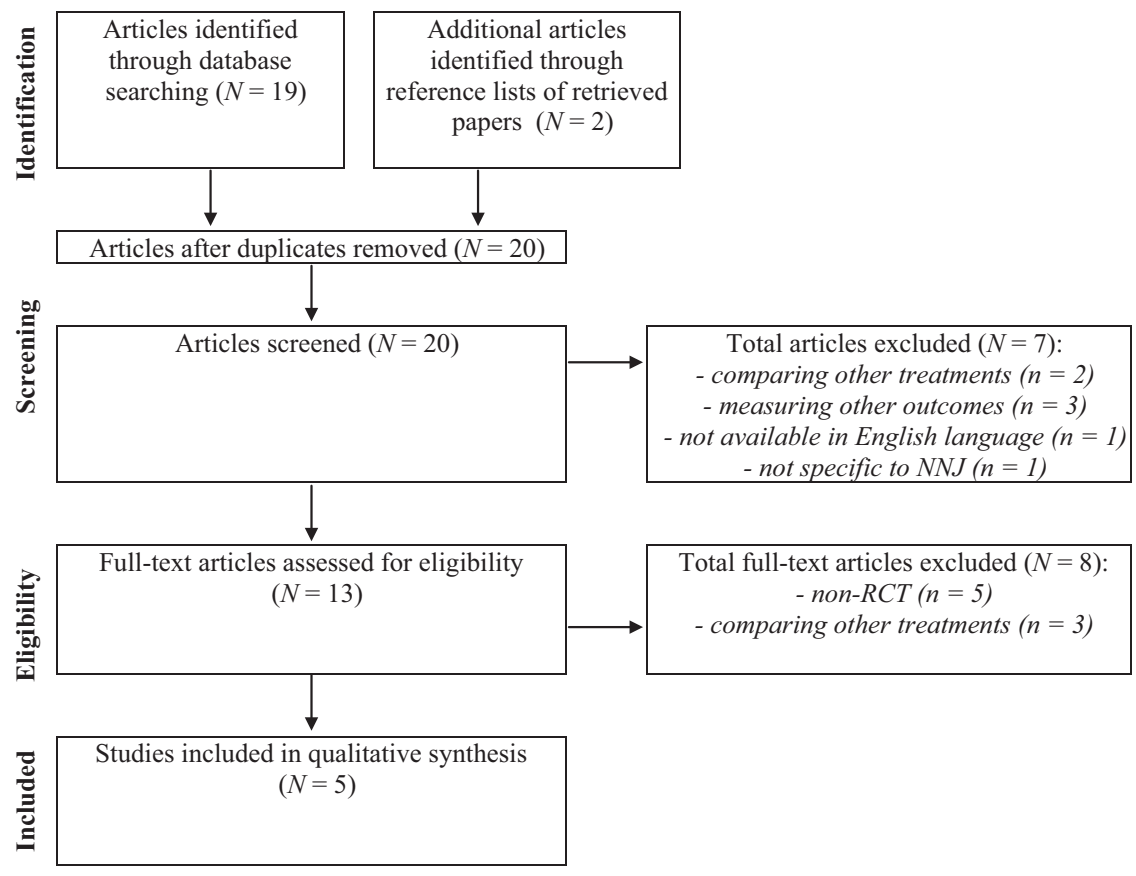

Figure 1 The Preferred Reporting Items for Systematic Reviews and Meta-Analyses 2009 flow diagram. 
aid in systematic reviews to appraise RCTs. It encompasses 11 domains as shown in Table 2. These reviewed articles were expected to fulfill the criteria as listed in this scale to enable them to be ranked high quality. A point was awarded only if the criterion had been clearly explained in the report. Violation of the fulfillment may result in the failure to accumulate points. Out of 10 points, papers with six points and above were considered moderate to high quality trials whilst those with five points and below were rated low quality trials (Centre of Evidence-Based Physiotherapy 2013). The first item, in which eligibility criteria ought to be specified, was excluded from the point allocation because it denoted the external validity of the findings. Other parameters to gain a point include the fulfillment of randomisation, concealed allocation, comparability between groups, blinding of all subjects, therapists and assessors, attrition rate of not more than $15 \%$, analysis by intention-to-treat, reporting of between-group results as well as the reporting results in point measures and measures of variability.

\section{Results}

Several components have been highlighted in evaluating and reporting the outcomes of this systematic review. Study selection, data extraction and quality assessments processes to appraise the findings of each paper were carried out. In line with the objectives of this review, two main components will be discussed in this section, namely the appraisal and findings of selected studies.

Table 2 The Physiotherapy Evidence Database scale

1. Eligibility criteria were specified

2. Subjects were randomly allocated to groups

3. Allocation was concealed

4. The groups were similar at baseline regarding the most important prognostic indicators

5. There was blinding of all subjects

6. There was blinding of all therapists who administered the therapy

7. There was blinding of all assessors who measured at least one key outcome

8. Measurements of at least one key outcome were obtained from more than $85 \%$ of the subjects initially allocated to groups

9. All subjects for whom outcome measurements were available received the treatment or control condition as allocated, or where this was not the case, data for at least one key outcome were analysed by 'intention to treat'

10. The results of between-group statistical comparisons are reported for at least one key outcome

11. The study provides both point measurements and measurements of variability for at least one key outcome

\section{Qualitative appraisal for selected papers}

\section{Evaluation via assessment tool}

Among the five studies assessed using the PEDro assessment tool, only one study was deemed of quality with the point of 7. On the other hand, the other four papers were awarded below six points as shown in Table 3 .

As depicted in Table 3, all five studies possess external validity by clearly specifying the eligibility criteria. Likewise, nonsignificant baseline regarding important prognostic indicators across groups, between-group outcome measures, point measures and measures of variability were reported in the five papers. The sufficiency in the reporting of statistical data enabled their results to be interpretable (criteria 10 and 11). Despite the fact that randomisation and concealed allocation are the most important elements in RCT, one paper gave a very confusing description on the randomisation and concealment processes (Mohammadzadeh et al. 2004). The unclear statement is quoted as follows:

After parental permission, twenty- five babies (turning group) were randomly changed from supine to prone position every $150 \mathrm{~min}-$ utes according to Shinwell's study followed by a break of 30 minutes for feeding and routine nursing care. (Mohammadzadeh et al. 2004, p. 228)

The other four trials (Chen et al. 2002, Shinwell et al. 2002, Mohammadzadeh et al. 2004, Donneborg et al. 2010) have otherwise indicated both the randomisation and concealment processes.

Blinding of subjects, therapists and assessors was not done in all these eligible trials. Having said so, Bhethanabhotla et al. (2013) did generally explain in their report concerning blinding:

Owing to obvious nature of intervention, blinding was not possible in our study. (Bhethanabhotla et al. 2013, p. 2)

Amid the five studies, one (Bhethanabhotla et al. 2013) evidently analysed the key outcome of all subjects initially allocated to groups. There was no loss of follow up. Nevertheless, the other four trials (Chen et al. 2002, Shinwell et al. 2002, Mohammadzadeh et al. 2004, Donneborg et al. 2010) did not mention nor provide adequate data to prove that there was $<15 \%$ of drop-out rate. Similarly, Bhethanabhotla et al. (2013) performed the statistical analysis by intention to treat whilst the other four trials (Chen et al. 2002, Shinwell et al. 2002, Mohammadzadeh et al. 2004, Donneborg et al. 2010) failed to specify their intention to treat in their reports.

The reporting of RCT conducted by Bhethanabhotla et al. (2013) was of good quality compare to the others 
Table 3 Qualitative evaluation of RCT using PEDro assessment tool

\begin{tabular}{|c|c|c|c|c|c|c|c|c|c|c|c|c|}
\hline Author & (1) & $(2)$ & (3) & (4) & $(5)$ & (6) & (7) & (8) & (9) & $(10)$ & $(11)$ & Total \\
\hline Chen et al. (2002) & レ & レ & レ & レ & $x$ & $x$ & $x$ & $x$ & $x$ & レ & $\boldsymbol{}$ & 5 \\
\hline Shinwell et al. (2002) & $\boldsymbol{}$ & レ & レ & レ & $x$ & $x$ & $x$ & $x$ & $x$ & レ & レ & 5 \\
\hline Mohammadzadeh et al. (2004) & $\boldsymbol{}$ & $x$ & $x$ & $レ$ & $x$ & $x$ & $x$ & $x$ & $x$ & レ & レ & 3 \\
\hline Donneborg et al. (2010) & 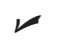 & レ & $\boldsymbol{\nu}$ & 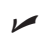 & $x$ & $x$ & $x$ & $x$ & $x$ & レ & 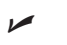 & 5 \\
\hline Bhethanabhotla et al. (2013) & 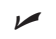 & レ & $\boldsymbol{V}$ & 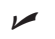 & $x$ & $x$ & $x$ & $\boldsymbol{V}$ & 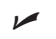 & $\boldsymbol{V}$ & $\boldsymbol{}$ & 7 \\
\hline
\end{tabular}

PEDro, Physiotherapy Evidence Database; RCT, randomized controlled trials; $\boldsymbol{\nu}$, criterion clearly explained; $\boldsymbol{x}$, criterion not clearly explained; (1), eligibility criteria; (2), random allocation; (3), concealed allocation; (4), baseline similar across groups; (5), subjects blinding; (6), therapists blinding; (7), assessors blinding; (8), less than 15\% drop-out rate; (9), analysis by 'intention to treat'; (10), between-group outcome measurement; (11), both point measures and measures of variability.

(Chen et al. 2002, Shinwell et al. 2002, Donneborg et al. 2010). Based on the PEDro scale, Bhethanabhotla et al. (2013) was likely to be internally valid as more than half of the criteria from amongst criteria $2-9$ had been clearly reported. Furthermore, the authors did acknowledge that blinding was not possible, although the specifics were not mentioned. In contrast, the rigor of study carried out by Mohammadzadeh et al. (2004) is questionable because the reporting was rather unclear and incomplete. Therefore, this trial may not be showing significant treatment effects (Maher et al. 2003).

\section{Evaluation on characteristics of papers}

Demographic and baseline characteristics. Table 4 summarises the characteristics of respective trials included in the review. A total of 340 newborns had been recruited for the review. The demographic and baseline characteristics were studied and analysed. According to the Paediatric Protocols for Malaysian Hospitals, there are numerous risk factors that heighten the TSB levels (Ismail et al. 2012). They include prematurity, small for gestational age as well as the other causes described in Background. Although one study (Donneborg et al. 2010) stated the inclusion criteria, demographic and clinical data of the patients recruited, the authors did not show the significant values across the groups for demographic and baseline data. Since both groups may not be comparable, confounding factors may alter the results of this particular trial and are doubtfully valid.

Majority of the studies $(n=4)$ (Chen et al. 2002, Shinwell et al. 2002, Mohammadzadeh et al. 2004, Bhethanabhotla et al. 2013) found no difference across groups for gestational age and birth weight. Four studies have excluded hemolytic conditions (Chen et al. 2002, Mohammadzadeh etal. 2004, Donneborg et al. 2010, Bhethanabhotla et al. 2013), whereas one paper established nonsignificant difference between groups on the positive Coombs' test (Shinwell et al. 2002). There were two studies
(Chen et al. 2002, Mohammadzadeh et al. 2004) that excluded neonates with cephalhematoma and one that excluded metabolic diseases (Mohammadzadeh et al. 2004). All studies but one (Donneborg et al. 2010) stated their exclusions of newborn with congenital anomalies during recruitment. Besides Mohammadzadeh et al. (2004), None of the trials accounted for their exclusions of neonates with sepsis. Nonetheless, three papers (Chen et al. 2002, Mohammadzadeh et al. 2004, Donneborg et al. 2010) vaguely mentioned about recruiting newborns who were healthy and clinically well.

As the severity of $\mathrm{NNJ}$ and the serum bilirubin reduction relies also on G6PD status, one study (Shinwell et al. 2002) did not report its exclusion on neonates with G6PD deficiency and neither did the authors run significant tests to compare G6PD status between two intervention groups. The other trials have either excluded such cases (Chen et al. 2002, Mohammadzadeh et al. 2004, Donneborg et al. 2010) or proven G6PD deficiency insignificant across groups (Bhethanabhotla et al. 2013). Forty percent of the included papers $(n=2)$ (Mohammadzadeh et al. 2004, Bhethanabhotla et al. 2013) recruited only exclusively breastfed newborns whilst two researches (Chen et al. 2002, Shinwell et al. 2002) found the varying feeds between two groups of neonates insignificant. One study (Donneborg et al. 2010) neither specifies feeding patterns of the sample nor does it provide significant values.

Likewise, hematocrit (HCT) or packed cell volume (PCV) levels, which help to diagnose polycythemia, were not reported or compared between groups in two studies (Chen et al. 2002, Donneborg et al. 2010). Polycythemia may leave the newborns clinically asymptomatic (WHO Collaborating Center for Training \& Research in Newborn Care 2007) but affects TSB level. One trial has proven that PCV levels between two groups are insignificant (Bhethanabhotla et al. 2013) while two other researches (Shinwell et al. 2002, Mohammadzadeh et al. 2004) reported that the HCT levels across groups showed no significant difference. 
Table 4 Characteristics of reviewed trials

\begin{tabular}{|c|c|c|c|c|}
\hline Study & $\begin{array}{l}\text { Sample } \\
\text { size }\end{array}$ & Intervention group 1 & Intervention group 2 & Study findings \\
\hline Chen et al. (2002) & $n=48$ & $\begin{array}{l}n=24 \\
\text { Fixed-position } \\
\text { group (FPG): } \\
\text { Received phototherapy } \\
\text { in supine position }\end{array}$ & $\begin{array}{l}n=27 \\
\text { Position-changing } \\
\text { group (PCG): } \\
\text { Received phototherapy by } \\
\text { rotating position from supine } \\
\text { to prone and vice versa every } \\
\text { two hours }\end{array}$ & $\begin{array}{l}\text { Rate of bilirubin reduction }(\mathrm{mg} / \mathrm{dl} / \mathrm{h}) \text { : } \\
\text { FPG }=0 \cdot 14(\mathrm{SD}=0.06) \\
\text { PCG }=0 \cdot 14(\mathrm{SD}=0.05) \\
p=0.89(\text { no difference }) \\
\text { Bilirubin decline at first } \\
24 \text { hours }(\%) \text { : } \\
\text { FPG: } 24.0(\mathrm{SD}=9.5) \\
\text { PCG: } 26 \cdot 0(\mathrm{SD}=9 \cdot 7) \\
p=0.47(\text { no difference) } \\
\text { Bilirubin decline at second } \\
24 \text { hours }(\%): \\
\text { FPG: } 19.1(\mathrm{SD}=7.3) \\
\text { PCG: } 17.4(\mathrm{SD}=8 \cdot 5) \\
p=0.54(\text { no difference })\end{array}$ \\
\hline $\begin{array}{l}\text { Shinwell } \\
\text { et al. (2002) }\end{array}$ & $n=30$ & $\begin{array}{l}n=14 \\
\text { Turned group (TG): } \\
\text { Supine or prone position } \\
\text { alternately for } 150 \text { minutes. Each } \\
\text { position is followed by } 30 \text { minutes } \\
\text { of feeding and routine nursing care }\end{array}$ & $\begin{array}{l}n=16 \\
\text { Supine group (SG): } \\
\text { Only in supine position }\end{array}$ & $\begin{array}{l}\text { Drop of serum bilirubin during first } \\
24 \pm 3 \text { hours from initial value }(\%) \text { : } \\
\mathrm{TG}=21(\mathrm{SD}=10) \\
\mathrm{SG}=29(\mathrm{SD}=8) \\
p=0.024 \text { (significant difference) } \\
\text { Duration of phototherapy (hours): } \\
\mathrm{TG}=40(\mathrm{SD}=15) \\
\mathrm{SG}=28(\mathrm{SD}=9) \\
p=0.03 \text { (significant difference) }\end{array}$ \\
\hline $\begin{array}{l}\text { Mohammadzadeh } \\
\text { et al. }(2004)\end{array}$ & $n=50$ & $\begin{array}{l}n=25 \\
\text { Supine group (SG): Supine } \\
\text { position throughout study period }\end{array}$ & $\begin{array}{l}n=25 \\
\text { Turned group (TG): Supine } \\
\text { to prone } \\
\text { position every } 150 \text { minutes } \\
\text { followed by a break of the } \\
30 \text { minutes for feeding and } \\
\text { nursing care }\end{array}$ & $\begin{array}{l}\text { Bilirubin at } 12 \text { hours after start of } \\
\text { phototherapy }(\mathrm{mg} / \mathrm{dl}) \text { : } \\
\mathrm{SG}=\text { mean } 14.8(\mathrm{SD}=2 \cdot 5) \\
\mathrm{TG}=\text { mean } 15 \cdot 2(\mathrm{SD}=1 \cdot 8) \\
p=0.58(\text { no difference) } \\
\text { Bilirubin at } 24 \text { hours after start of } \\
\text { phototherapy }(\mathrm{mg} / \mathrm{dl}): \\
\mathrm{SG}=\text { mean } 12 \cdot 2(\mathrm{SD}=2 \cdot 7) \\
\mathrm{TG}=\text { mean } 12 \cdot 1(\mathrm{SD}=2 \cdot 0) \\
p=0 \cdot 74(\text { no difference) } \\
\text { Bilirubin at } 48 \text { hours after start of } \\
\text { phototherapy }(\mathrm{mg} / \mathrm{dl}): \\
\mathrm{SG}=\text { mean } 9.5(\mathrm{SD}=2 \cdot 4) \\
\mathrm{TG}=\text { mean } 9 \cdot 6(\mathrm{SD}=2 \cdot 1) \\
p=0.93(\text { no difference })\end{array}$ \\
\hline $\begin{array}{l}\text { Donneborg } \\
\text { et al. (2010) }\end{array}$ & $n=112$ & $\begin{array}{l}n=59 \\
\text { Alternating group (AG): } \\
\text { Supine to prone and vice versa } \\
\text { every third hour }\end{array}$ & $\begin{array}{l}n=53 \\
\text { Supine group (SG): } \\
\text { Exclusively in supine } \\
\text { position }\end{array}$ & $\begin{array}{l}\text { Decrease of TSB after } 12 \text { hours of } \\
\text { phototherapy }(\%): \\
\text { AG }=\text { mean } 32 \\
(95 \% \mathrm{CI}=30,34) \\
\mathrm{SG}=\text { mean } 32 \\
(95 \% \mathrm{CI}=30,35) \\
p=0.86 \text { (no difference) } \\
\text { Decrease of TSB after } 24 \text { hours of } \\
\text { phototherapy }(\%): \\
\text { AG }=\text { mean } 49 \\
(95 \% \mathrm{CI}=47,51) \\
\mathrm{SG}=\text { mean } 50 \\
(95 \% \mathrm{CI}=47,53) \\
p=0.66 \text { (no difference) }\end{array}$ \\
\hline
\end{tabular}


Table 4 (Continued)

\begin{tabular}{|c|c|c|c|c|}
\hline Study & $\begin{array}{l}\text { Sample } \\
\text { size }\end{array}$ & Intervention group 1 & Intervention group 2 & Study findings \\
\hline $\begin{array}{l}\text { Bhethanabhotla } \\
\text { et al. (2013) }\end{array}$ & $n=100$ & $\begin{array}{l}n=54 \\
\text { Supine group (SG): } \\
\text { No change in position }\end{array}$ & $\begin{array}{l}n=46 \\
\text { Turning group (TG): Supine } \\
\text { to prone and vice versa every } \\
\text { two-hourly }\end{array}$ & $\begin{array}{l}\text { Rate of TSB reduction }\left(m g d l b^{-1}\right) \text { : } \\
\mathrm{SG}=0 \cdot 20(\mathrm{SD}=0 \cdot 1) \\
\mathrm{TG}=0.22(\mathrm{SD}=0 \cdot 1) \\
\text { Mean difference }(95 \% \mathrm{CI})=-0.02 \\
(-0.06,0 \cdot 02) \\
p=0.34 \text { (no difference) } \\
\text { Duration of phototherapy (hours): } \\
\mathrm{SG}=25 \cdot 5(\mathrm{SD}=8) \\
\mathrm{TG}=24.8(\mathrm{SD}=5) \\
\text { Mean difference }(95 \% \mathrm{CI})=0.7 \\
(-2 \cdot 03,3.44) \\
p=0.60 \text { (no difference) }\end{array}$ \\
\hline
\end{tabular}

SD, standard deviation; CI, confidence interval; TSB, total serum bilirubin concentration.

Irradiance from a phototherapy system is one of the important factors in bringing down unconjugated bilirubin and is affected by the types of lamp and distance of light source from surface of the newborns (Cameron \& Hart 2005). Three of the studies (Chen et al. 2002, Shinwell et al. 2002, Bhethanabhotla et al. 2013) revealed, standardised and controlled the irradiance to be comparable across groups while the other two studies (Mohammadzadeh et al. 2004, Donneborg et al. 2010) failed to mention about this. Having said so, irradiance of the phototherapy unit used in two of the trials (Chen et al. 2002, Shinwell et al. 2002) was found to be lower than the minimum irradiance of $15 \mu \mathrm{W} / \mathrm{cm}^{2} / \mathrm{nm}$ as outlined in the Paediatric Protocols for Malaysian Hospitals (Ismail et al. 2012).

Methods. In the turning and alternating group in the three trials (Chen et al. 2002, Donneborg et al. 2010, Bhethanabhotla et al. 2013), each position lasted for two hours before the newborns were placed in another position. Meanwhile, newborns in the turned groups assumed in the same position for 2.5 hours in the other two trials (Shinwell et al. 2002, Mohammadzadeh et al. 2004). All researches exposed the newborns in the same manner, which is keeping them naked except for diapers and eye pads. Likewise, all the studies $(n=5)$ reported the duration when newborns were removed from phototherapy for feeding and routine nursing care. Among the five trials, one (Bhethanabhotla et al. 2013) has meticulously designed a monitoring chart for recording the duration of phototherapy. Furthermore, compliance with the duration was closely monitored and scrutinised by the investigators. Two studies (Chen et al. 2002, Mohammadzadeh et al. 2004) reported the total duration of phototherapy in both groups and have proved it to be similar between groups. On the other hand, the remaining studies $(n=2)$ (Shinwell et al. 2002, Donneborg et al. 2010) did not discuss on the compliance with the duration of phototherapy.

In obtaining the sample size, most of the researches $(n=3)$ (Shinwell et al. 2002, Donneborg et al. 2010, Bhethanabhotla et al. 2013) have described both the power and alpha values. Bhethanabhotla et al. (2013) calculated with an alpha $(\alpha)$ of 0.05 and power of $90 \%$ while Shinwell et al. (2002) and Donneborg et al. (2010) with $\alpha$ of 0.05 and power of $80 \%$. However, $60 \%$ of the studies $(n=3)$ (Chen et al. 2002, Shinwell et al. 2002, Mohammadzadeh et al. 2004) have sample sizes of $<100$ and may be subject to a lack of statistical power to detect treatment difference. Hence, these results are endangered with type II error (Rosner 2006).

A minority of the study $(n=1)$ (Bhethanabhotla et al. 2013) clearly outlined flow of the trial as recommended by the Consolidated Standards of Reporting Trials (CONSORT) guidelines (Schulz et al. 2010). This study also utilised stratified randomisation to optimise similarity in both groups.

Statistical analyses and outcome measures. Among the five trials appraised, $40 \%(n=2)$ (Mohammadzadeh et al. 2004, Donneborg et al. 2010) were found to have hazily reported statistical tests used to analyse the different types of variables. Outcome measures were rather alike among the studies. One paper in particular differentiated the outcome measures into primary and secondary (Bhethanabhotla et al. 2013). The primary outcome is the duration of phototherapy while the secondary outcome is the rate of fall of bilirubin. Eighty percent $(n=4)$ of the trials nevertheless, did not distinguish the primacy of the outcome measures. Outcome measures such as the decrease of TSB 
(Chen et al. 2002, Shinwell et al. 2002, Mohammadzadeh et al. 2004), and the drop in serum bilirubin values as well as the duration of phototherapy (Donneborg et al. 2010) have been reported. Clinical side effects of phototherapy were reported in most of the studies $(n=3)$ (Mohammadzadeh et al. 2004, Donneborg et al. 2010, Bhethanabhotla et al. 2013).

In conclusion, following the analyses based on the PEDro scale and the elaborated critiques on characteristics of the papers, it was discovered that there was only one paper (Bhethanabhotla et al. 2013) deemed of high quality with total points of 7 .

\section{Findings of reviewed trials}

Five randomised clinical trials have been included in this systematic review. All five papers (Chen et al. 2002, Shinwell et al. 2002, Mohammadzadeh et al. 2004, Donneborg et al. 2010, Bhethanabhotla et al. 2013) unearthed that turning newborns during phototherapy did not significantly decrease the TSB levels and the duration of phototherapy. Four studies (Chen et al. 2002, Mohammadzadeh et al. 2004, Donneborg et al. 2010, Bhethanabhotla et al. 2013) reported no difference between turning and laying the neonates supine under phototherapy in terms of rate of TSB reduction and the duration of phototherapy. There was however one study (Shinwell et al. 2002) which discovered that putting the newborns supine throughout phototherapy was more therapeutic than alternating the position within two to three hours. However, its small sample size $(n=30)$ may alter the level of precision and its accuracy in representing the population. No study has proven that turning newborns two to three hourly during phototherapy was superior to placing the newborns supine throughout the phototherapy.

From the findings reported in all five reviewed articles, there was no evidence supporting the hypothesis that turning the jaundiced neonates at two to three hourly throughout phototherapy could hasten the reduction of TSB levels. Therefore, it can be concluded from these articles that having jaundiced neonates receiving phototherapy in a supine position is as effective as alternating their positions.

\section{Discussion}

All five appraised papers have reported that turning newborns during phototherapy did not significantly decrease the TSB levels and the duration of phototherapy. Four studies reported no difference between turning vs. laying the neonates supine under phototherapy whilst one study (Shin- well et al. 2002) discovered that putting the newborns supine throughout phototherapy was more therapeutic than alternating the position every two to three hours. No study has proven that turning newborns two to three hourly during phototherapy was superior to placing the newborns supine throughout phototherapy.

External validity of these studies relies entirely on the eligibility criteria. The study results can be made generalisable to term neonates with hyperbilirubinemia because the studied sample composed of newborns with gestational age above 37 weeks. The treatment effect however, ought to be applied cautiously to the moderate or late preterm neonates $(32-<37$ weeks) as only two studies have recruited this group of newborns as their sample (Donneborg et al. 2010, Bhethanabhotla et al. 2013).

Findings from these trials were opposed to the theory generated by Vogl et al. (1978) and Lau et al. (1984) on the kinetic mechanism of serum bilirubin, in which the blanched surface would be reloaded with bilirubin when the exposed surface has been changed and alternated with the bilirubin-loaded side. Based on their models, changing the position of jaundice neonates or implementing intermittent phototherapy would hasten the drop of TSB by optimising photoisomerisation. Nonetheless, the findings from these reviewed trials did not support the superiority of alternating position in bringing down the serum bilirubin among jaundiced neonates nursed under single phototherapy.

Direct comparison of the outcome measures may not be appropriate as the model of phototherapy unit, radiometer and laboratory machines running blood specimens vary across studies. These apparatus, nonetheless, have been standardised in each study.

As explained in one of the studies, blinding of the participants, nurses and assessors was not feasible due to the nature of the study. In a study determining the efficacy of another intervention in hastening the drop of bilirubin level for NNJ nursed under conventional phototherapy, measures had been taken to blind the laboratory technologists from the intervention (Abd Hamid et al. 2013). Therefore, blinding could be done to a certain extend and ought to be attempted in as many health care personnel as possible with a view to minimise bias that may affect the result.

It has been documented that prematurity, low birth weight, small for gestational age, breast milk, haemolytic conditions, $\mathrm{ABO} /$ rhesus incompatibility, medical/surgical/ congenital co-morbidity, jaundice within 24 hours of life, G6PD deficiency, siblings with severe NNJ, instrumental/ induced delivery, antenatal co-morbidities, prior phototherapy and medication such as phenobarbitone or 
vitamin $\mathrm{K}$ affects the severity of hyperbilirubinemia among neonates (Carswell et al. 1972, Kumar et al. 2002, Maisels \& Kring 2002, Hockenberry \& Wilson 2011, Ismail et al. 2012,). It is hence, crucial to ensure that these demographic and baseline data have been acknowledged, well-controlled and proven nonsignificant across groups to avoid confounding factors that influence the validity and reliability of the findings. There was not one study that has completely controlled these extraneous factors although some have managed most of these factors. Thus, a high quality trial considering all these factors is called for in strengthening the evidence and rigor of research in this area.

\section{Limitations}

One of the pitfalls of this systematic review is the language barrier that prohibited the retrieval and understanding of journal articles written in languages other than English language. As the articles reviewed were restricted to English language only, similar studies reported in the other languages were consequently not appraised.

\section{Conclusion}

Utilising the PEDro assessment tool, one out of five studies was deemed of high quality. Subsequently, based on that particular high quality randomised controlled trial in majority of the papers, keeping the jaundiced newborns in supine position throughout phototherapy was as effective as turning them because these trials have found no difference in the rate of TSB reduction and length of hospital stay of these neonates. Furthermore, in view of the fact that guidelines such as the Paediatric Protocol for Malaysian Hospital (3rd ed.) (Ismail et al. 2012) have removed the two-hourly turning in the care of neonates under phototherapy in the latest edition, coupled with the National Institute for Health and Care Excellence (NICE) (2010) and Queensland Maternity and Neonatal Clinical Guidelines Program (2009) of placing newborns supine under phototherapy at all times according to the safe infant sleeping protocol, the best position jaundiced neonates should assumed is the supine position and it is not necessary to alternate positions on a two to three hourly basis when conventional phototherapy is delivered.

\section{Relevance to clinical practice}

Turning a child every two hours used to be one of the nursing interventions delivered to neonates who underwent phototherapy in some local hospitals. To date, according to a worldwide survey, many departments and hospitals are still practicing two-hourly turning in managing neonates with jaundice (Mohammadzadeh et al. 2004, Hansen 2012). It has been reported that the newborns developed NNJ in their first week of life, in which $60 \%$ were term whilst $80 \%$ were preterm neonates (Maisels \& McDonagh 2008, Welsh 2010). With the shocking percentage, nurses' workload will inevitably be increased by having to turn the newborn every two to three hourly. Nevertheless, the outcome of this systematic review has revealed otherwise. Turning the child every two to three hours is as effective as putting the child in a supine position. Hence, it is unnecessary to alternate the position of these neonates. The findings from this systematic review have contributed widely to the nursing practice by cutting down unnecessary and redundant intervention. With the nurses' workload lightened, the quality of nursing care will most likely be improved as they can focus more on other necessary care, resulting in better patient outcomes.

\section{Disclosure}

The authors have confirmed that all authors meet the ICMJE criteria for authorship credit (www.icmje.org/ethical_1author.html), as follows: (1) substantial contributions to conception and design of, or acquisition of data or analysis and interpretation of data, (2) drafting the article or revising it critically for important intellectual content, and (3) final approval of the version to be published.

\section{References}

Abd Hamid IJ, M Iyen MI, Ibrahim NR, Abd Majid N, Ramli N \& Van Rostenberghe H (2013) Randomised controlled trial of single phototherapy with reflecting curtains versus double phototherapy in term newborns with hyperbilirubinaemia. Journal of Paediatrics and Child Health 49, 375-379.
Bhethanabhotla S, Thukral A, Sankar MJ, Paul VK \& Deorari AK (2013) Effect of position of infant during phototherapy in management of hyperbilirubinemia in late preterm and term neonates: a randomized controlled trial. Journal of Perinatology 2013, $1-5$.
Cameron R \& Hart G (2005) The importance of irradiance and area in neonatal phototherapy. Archives of Disease in Childhood, Fetal and Neonatal Edition 90, 437-440.

Carswell F, Kerr MM \& Dunsmore IR (1972) Sequential trial of effect of phenobarbitone on serum bilirubin 
of preterm infants. Archives of Disease in Childhood 47, 621.

Centre of Evidence-Based Physiotherapy (2013) PEDro Statistics. PEDro Physiotherapy Evidence Database. Available at: http://www.pedro.org.au/ english/downloads/pedro-statistics/ (accessed 2 July 2013).

Chen CM, Liu SH, Lai CC, Hwang CC \& Hsu HH (2002) Changing position does not improve the efficacy of conventional phototherapy. Acta Paediatrica Taiwanica 43, 255-258.

Donneborg ML, Knudsen KB \& Ebbesen F (2010) Effect of infants' position on serum bilirubin level during conventional phototherapy. Acta Paediatrica 99, 1131-1134.

Hansen TWR (2012) Neonatal Jaundice. Emedicine Medscape. Available at: http://emedicine.medscape.com/article/ 974786-overview (accessed 5 July 2013).

Hockenberry MJ \& Wilson D (2011) Wong's Nursing Care of Infants and Children, 9th edn. Elsevier, Mosby, Canada.

Haji Muhammad Ismail HM, Ng HP \& Thomas T (2012) Paediatric Protocols for Malaysian Hospitals, 3rd edn. Ministry of Health of Malaysia, Malaysia.

Kumar R, Narang A, Kumar P \& Garewal G (2002) Phenobarbitone prophylaxis for neonatal jaundice in babies with birth weight 1000-1499 grams. Indian Pediatrics 39, 945.
Lau SP \& Fung KP (1984) Serum bilirubin kinetics in intermittent phototherapy of physiological jaundice. Archives of Disease in Childhood 59, 892-894.

Maher CG, Sherrington C, Herbert RD, Moseley AM \& Elkins M (2003) Reliability of the PEDro scale for rating quality of randomized controlled trials. Physical Therapy 83, 713-721.

Maisels MJ \& Kring E (2002) Rebound in serum bilirubin level following intensive phototherapy. Archives of Pediatrics \& Adolescent Medicine 156, 669672.

Maisels MJ \& McDonagh AF (2008) Phototherapy for neonatal jaundice. The New England Journal of Medicine 358(9), 920-928.

Mohammadzadeh A, Bostani Z \& Jafarnejad F (2004) Supine versus turning position on bilirubin level during phototherapy in healthy term jaundiced neonates. Medical Journal of the Islamic Republic of Iran 18, 227-230.

Moher D, Liberati A, Tetzlaff J, Altman DG \& The PRISMA Group (2009) Preferred reporting items for systematic reviews and meta-analyses: the PRISMA statement. Journal of Clinical Epidemiology 62, 1006-1012.

National Institute for Health and Care Excellence (NICE) (2010) Recognition and Treatment of Neonatal Jaundice. Neonatal Jaundice (CG98). Available at: http://guidance.nice.org.uk/cg98 (accessed 14 May 2014).
Queensland Maternity and Neonatal Clinical Guidelines Program (2009) Neonatal Jaundice: Prevention, Assessment and Management. Queensland Maternity and Neonatal Clinical Guideline. Available at: http://www.health.qld. gov.au/qcg/documents/g_jaundice5-1. pdf (accessed 14 May 2014).

Rosner B (2006) Fundamentals of Biostatistics, 6th edn. Thomson Brooks/Cole, Boston MA.

Schulz KF, Altman DG \& Moher D (2010) CONSORT 2010 statement: updated guidelines for reporting parallel group randomised trials. BioMed Central Medicine 8, 1-9.

Shinwell ES, Sciaky Y \& Karplus M (2002) Effect of position change on bilirubin levels during phototherapy. Journal of Perinatology 22, 226-229.

Vogl TP, Hegyi T, Hiatt IM, Polin RA \& Indyk L (1978) Intermediate phototherapy in the treatment of jaundice in the premature infant. The Journal of Pediatrics 92, 627-630.

Welsh A (2010) Neonatal Jaundice: Clinical Guideline. Royal College of Obstetricians and Gynaecologists, London.

World Health Organization (WHO) Collaborating Center for Training and Research in Newborn Care (2007) Polycythemia in the Newborn. Available at: http://www.newbornwhocc. org/ (accessed 2 September 2013).

The Journal of Clinical Nursing (JCN) is an international, peer reviewed journal that aims to promote a high standard of clinically related scholarship which supports the practice and discipline of nursing.

For further information and full author guidelines, please visit JCN on the Wiley Online Library website: http:// wileyonlinelibrary.com/journal/jocn

\section{Reasons to submit your paper to JCN:}

High-impact forum: one of the world's most cited nursing journals, with an impact factor of 1.316 - ranked 21/101 (Nursing (Social Science)) and 25/103 Nursing (Science) in the 2012 Journal Citation Reports ${ }^{\circledR}$ (Thomson Reuters, 2012).

One of the most read nursing journals in the world: over 1.9 million full text accesses in 2011 and accessible in over 8000 libraries worldwide (including over 3500 in developing countries with free or low cost access).

Early View: fully citable online publication ahead of inclusion in an issue.

Fast and easy online submission: online submission at http://mc.manuscriptcentral.com/jcnur.

Positive publishing experience: rapid double-blind peer review with constructive feedback.

Online Open: the option to make your article freely and openly accessible to non-subscribers upon publication in Wiley Online Library, as well as the option to deposit the article in your preferred archive. 